\title{
Gran Salvo. Faro interactivo para el Palacio Salvo
} Gran Salvo. Interactive lighthouse for the Palacio Salvo

\author{
Federico Lagomarsino \\ Universidad de la República \\ lagomarsino.federico@gmail.com \\ Fernando García Amen \\ Universidad de la República \\ efe@fadu.edu.uy
}

\begin{abstract}
This paper aims to expose a reflection on the impact created by the construction of the new lighthouse on the Palacio Salvo building. It focuses on the design process, fabrication and installation of this new object, focused in the use of light as a tool for communication and its interaction with society by adding different functions operated by apps.
\end{abstract}

Keywords: patrimonio; fabricación digital; luz; diseño paramétrico; acción urbana

\section{Introducción}

Este trabajo pretende generar una reflexión acerca del faro perdido del edificio más emblemático de la ciudad de Montevideo, el Palacio Salvo. Se trata de una construcción icónica y probablemente la más reconocida del skyline montevideano. La historia de esta pérdida, si bien está trazada por diversas aristas, es una de las tantas historias urbanas que moldean y prefiguran la identidad de la ciudad. Declarado patrimonio cultural nacional, el Palacio Salvo es un referente montevideano, y la ausencia del remate en su cúpula constituyó, por años, un vacío urbano que merecía ser completado. Originalmente construido en 1928, los relatos de su faro original son variados. Se dice que fue retirado a pedido de las fuerzas armadas para evitar confusiones en la logística portuaria, se dice también que el faro se encuentra oculto en la chacra de uno de sus antiguos dueños e incluso existen rumores que alegan que el faro nunca se colocó.

Gran Salvo es el nombre de este nuevo faro. Este elemento como tal es al mismo tiempo una estructura de remate sobre una cúpula y un dispositivo emisor de luz. En el caso abordado, se previó la concreción de ambas funciones en la producción de un objeto acabado, cuyo alcance tendrá una dimensión social valorable en el tiempo real, pero que sin dudas trascenderá también en el tiempo.

La gestión del proyecto inicia en el 2016 a través de la presentación de la propuesta al alcalde del Municipio B y luego a la directiva administrativa del edificio. Si bien el Palacio Salvo es un edificio que la ciudad lo siente como propio, en contraste con esta percepción, el edificio es de carácter privado y funciona como una sociedad anónima, con accionistas que compran porcentajes y adquieren superficies de la propiedad. Esta condición ha generado situaciones muy diversas dentro del edificio, generando contrastes sociales muy marcados que durante los últimos 30 años han deteriorado la imagen e infraestructura del edificio.
Recientemente, a través de la implementación de una nueva directiva a cargo del palacio, su gestión ha tomado otra dirección, buscando la recuperación patrimonial, la incorporación de programas culturales y turísticos con la finalidad de devolverle el esplendor perdido. Es este escenario que recibe al proyecto y que permite llevarlo adelante.

La idea inicial del proyecto era completar el negativo de la silueta y al mismo tiempo generar un contenido específico para el retorno de la luz de la ciudad, una luz que no debería ser decorativa, sino que pudiera vincularse a un mensaje, como lo haría un faro. Cabe destacar que inicialmente el proyecto se iba a desarrollar como una propuesta académica alojada por la Facultad de Arquitectura, pero finalmente se llevó adelante como un proyecto de carácter independiente debido a las complejidades y las responsabilidades profesionales y técnicas demandadas.

\section{Metodología}

En gran parte, el marco teórico de este proyecto se asienta sobre la doble inquietud de llevar adelante la ejecución de proyectos urbanos de impacto social, y la implementación de instalaciones de base tecnológica y artística a nivel urbano y/o arquitectónico.

El proyecto se nutre de dos visiones sobre los posibles futuros de las ciudades que provienen del mundo de la programación digital. La primer visión plantea un futuro convergente, donde se plantea que la relación del ser humano con su entorno se establecerá a través de un único dispositivo. El segundo futuro, el divergente, plantea que será el entorno el que responderá a nuestras acciones y podrá reaccionar hacia los ciudadanos, sin intermediarios. Estas dos visiones estuvieron presentes en el desarrollo del proyecto y sin duda la obra pretende interpelar estas visiones.

En términos de gestión, el reto de vencer trabas burocráticas, así como gestionar el proceso de cambio a través de la 
amortiguación del potencial rechazo que naturalmente todo cambio posee, fueron una parte esencial de este desafío. No obstante, la resolución de estas instancias contribuyó a promover el debate sobre el que se llevó a cabo la tarea, y a poner en el foco de discusión la pertinencia de la intervención, la utilización de las tecnologías digitales en la construcción, y la implementación de la interactividad en la arquitectura.

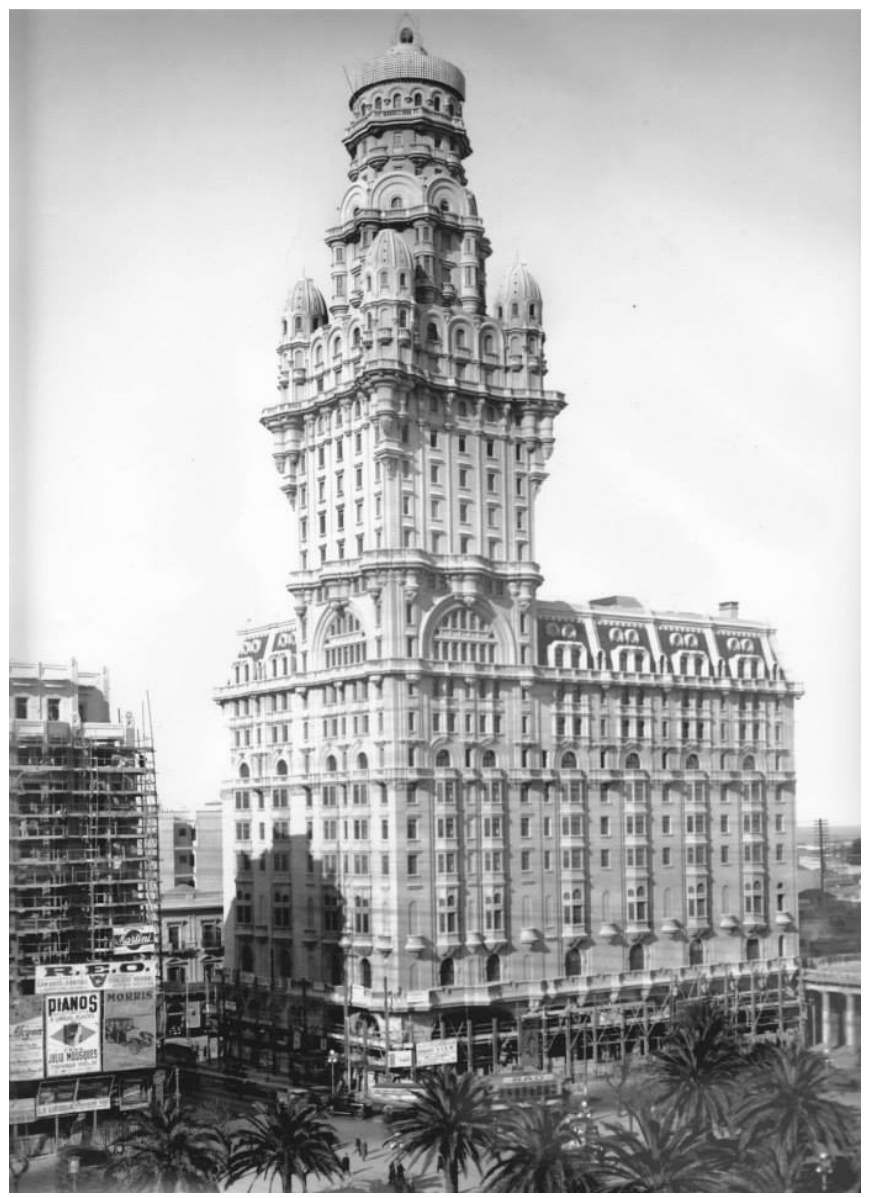

Figura 1: Imagen histórica del Palacio Salvo de Montevideo, inaugurado en 1928.

Sobre estas premisas, se conformó un grupo de trabajo conformado por arquitectos, ingenieros, constructores, programadores y estudiantes de grado.

Los desafíos tecnológicos para la formulación de una solución constructiva para el faro del Palacio Salvo de Montevideo, fueron los siguientes:

- Diseño paramétrico realizado en BIM.

- Involucramiento del manejo inteligente de la luz como un elemento central del proyecto;
- Habilitar la interacción con la sociedad a través de la comunicación de celebraciones y fechas especiales a través de los colores del faro.

- Generación de contenidos inteligentes, que toman información de bases de datos y los comunican a través de la luz.

- Habilitar la interacción del objeto con la sociedad a través de una app específica de control de luces y funcionalidad de las mismas.

En línea con los adelantos para "el internet de las cosas", donde objetos ordinarios son conectados a internet, el faro también pretende ser un aporte a esta tendencia desde la escala urbana.

La participación de la luz como elemento central del proyecto se definió en forma sustantiva con el apoyo del programador y los ingenieros en iluminación, y se implementó a través de un sistema led RGB y sus respectivos controladores. Su manejo es versátil y adaptable a distintos usos, cambios de color, intensidad y producción de contenidos, generando así un hardware con potencialidad de ser programado en distintos sentidos y con diferentes funcionalidades. Las luces encienden y se controlan a través de placas Raspberry Pi 3, constituyendo un elemento de muy bajo coste en comparación con la potencia lumínica y con las posibilidades que ofrecen en su uso, mientras que la interfaz es DALI.

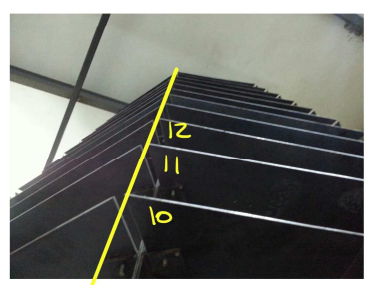

EXT

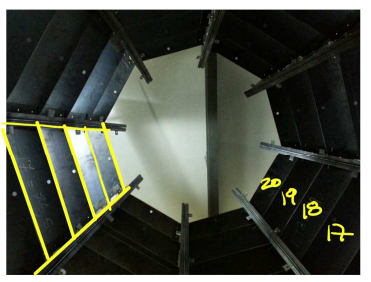

(NT

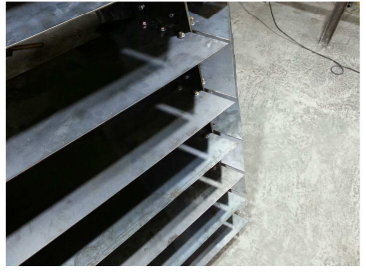

prenontase

No

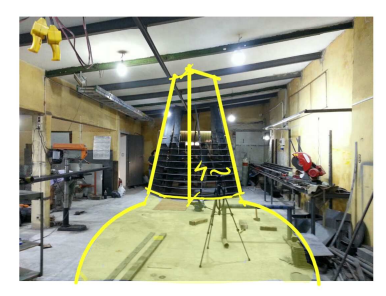

Figura 2: Diseño y producción en taller del objeto interactivo (el Faro), previo a su instalación. 

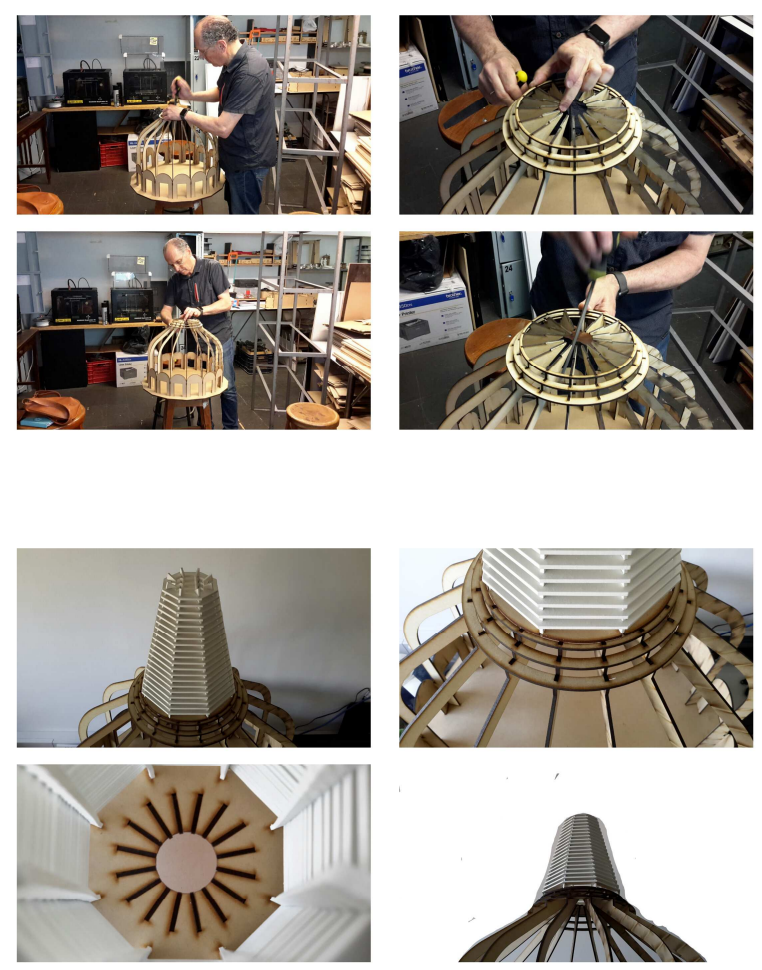

Figura 3: Maqueta de experimentación, en corte CNC, a escala. Pruebas realizadas en el LabFab de la Facultad de Arquitectura, Diseño y Urbanismo.

Inmediatamente luego de su inauguración el faro ya acompañó varias fechas y celebraciones especiales como, el día de la esclerosis múltiple, el día de la diversidad, aniversario del edificio Barolo y la semana del corazón.

Actualmente está en proceso la realización el primer contenido inteligente, la idea será comunicar el pronóstico del estado del tiempo a través de la luz, exclusivamente comunicar la lluvia utilizando el color azul, algo tan fundamental, primitivo y simple como el agua. Este contenido funcionará como base permanente e irá intercalando diferentes usos de la luz, como las celebraciones y futuras interacciones con apps.

Para el año 2018 se está planificando la producción de una app de control en poder ciudadano, que será la próxima etapa de este proyecto. Con esta finalidad se está planificando la realización de una hackatón para el desarrollo de la misma. Su funcionalidad será definida y probada con un grupo reducido de usuarios y luego liberada para el uso de la población en general.

El faro realizado comprende una estructura metálica, de base octogonal con $9 \mathrm{~m} 2$ de superficie y una altura final de $4 \mathrm{~m}$ sobre nivel de piso terminado existente. Llegando a los $105 \mathrm{~m}$ final del edificio.

Todos sus elementos fueron arenados y terminados con fondo epoxi y pintura poliuretánica blanco mate.
La fijación a la base se realizó a través de 8 platinas de acero de $2,5 \mathrm{~cm}$ de espesor, calidad A36, colocadas sobre un grouting de $4 \mathrm{~cm}$ y fijada a la base con varillas de acero roscado de $16 \mathrm{~mm}$, con anclaje químico y con platinas de traba en los sectores que la varilla atraviesa la losa.

Toda la estructura fue previamente realizada en taller, modulada para poder subirla al sitio a través del ascensor. Para el montaje se utilizaron bulones de $8 \times 1,25 \mathrm{~mm}$, tuercas con freno y arandelas. Se colocaron las celosías $(3.18 \mathrm{~mm})$ en cada una de las caras, colocadas con bulones a planchuelas de $15 \times 5 \mathrm{~cm}$ y espesor $6 \mathrm{~mm}$ soldadas a los pilares, hasta llegar a los primeros $2 \mathrm{~m}$. En la segunda etapa se agregaron los siguientes módulos de pilares hasta llegar a los $4 \mathrm{~m}$ de altura y luego las celosías correspondientes hasta completar 20 celosías en cada una de las 8 caras.

Se colocó a $20 \mathrm{~cm}$ sobre la última celosía un anillo de cobre $6 \mathrm{~mm}$ vinculado a dos bajadas a tierra existentes en el edificio previamente verificadas. Esta instalación actúa de captor para descargas atmosféricas y protege la instalación. El edificio no contaba con un pararrayos y estaba desprotegido.

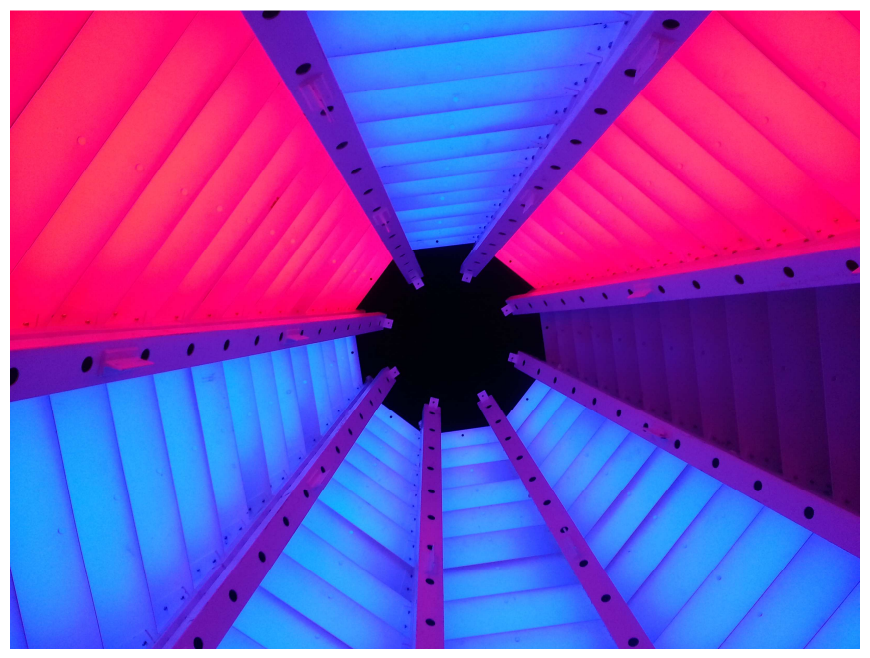

Figura 4: Imagen del Faro interactivo desde dentro, con la instalación lumínica ya operativa.

\section{Resultados obtenidos y esperados}

Sustantivamente, el aporte de este trabajo es que se conformó un objeto capaz de adquirir valor propio en tanto que unidad de diseño, pero capaz de coexistir en simbiosis con el edificio en el que se inserta, dado su valor patrimonial e histórico. Un elemento que actúa como simbionte con el proyecto original, aportándole valor urbano y a viceversa.

Asimismo, el objeto fabricado es accesible y con la previsión de ser socialmente interactivo, permitiendo diversidad de usos a través del manejo de los dispositivos de iluminación instalados. No está definido aun, si este nuevo faro, será una construcción con carácter provisorio o acaso permanente. Esto se definirá por el grado de aceptación social que se produzca, medido en el tiempo. 


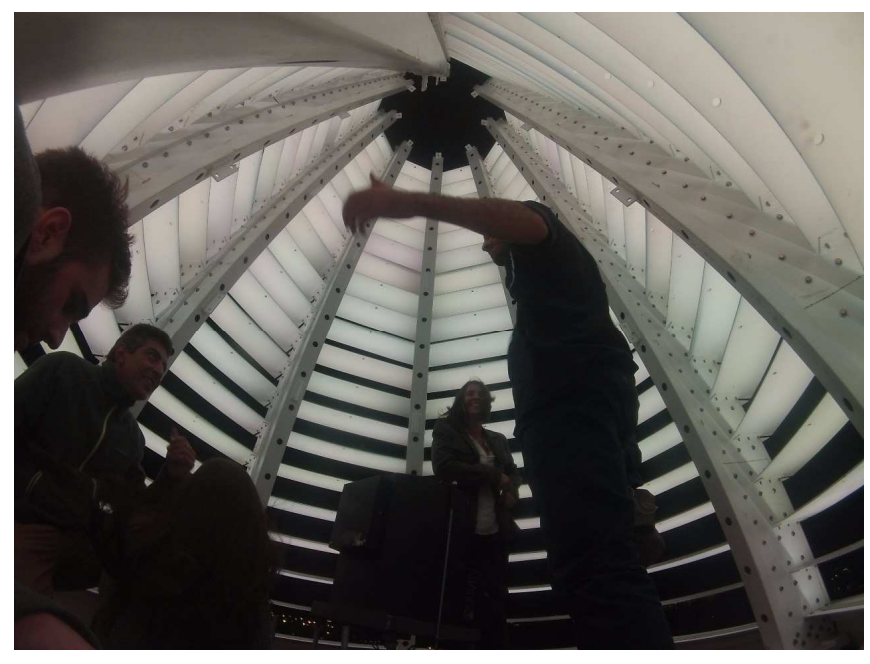

Figura 5: Equipo de trabajo durante visita nocturna.

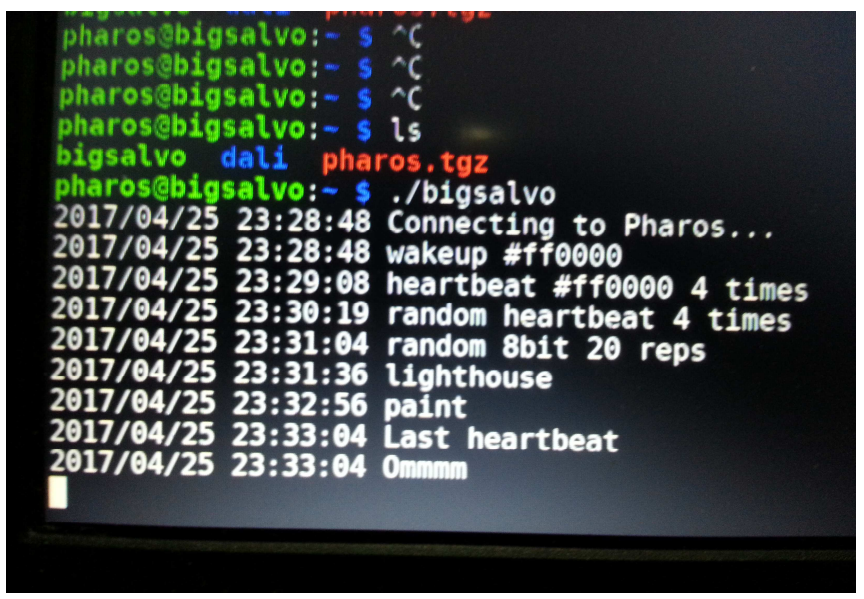

Figura 6: Código de pruebas, y primeras comunicaciones con el Faro interactivo.

En el momento de redacción de este texto, el objeto lleva pocos meses desde su inauguración, habiendo recogido decenas de notas de prensa, televisión y demás medios, denotando el alto impacto que en la población ha generado.

No obstante, habrá que realizar un seguimiento más exhaustivo y sostenido temporalmente, para medir, mediante encuestas, la valoración crítica por parte de la sociedad, que será en última instancia el juez del proyecto.

En cualquier caso, se espera que el objeto fabricado se constituya en un hito urbano capaz de transformar, desde su naturaleza contemporánea, la imagen de un edificio icónico para la ciudad.

\section{Debate}

La desaparición de la histórica cúpula y por consiguiente del remate en el diseño del Palacio Salvo de Montevideo, lejos de ser un tema exclusivo de los académicos, debe abordarse como un tópico social, pues atañe al patrimonio urbano y a la identidad de la ciudad.

La fabricación de faro, será sin dudas un aporte tangible a la discusión y a la toma de conciencia sobre la permanencia y el cambio en un edificio emblemático, desde la aplicación de las nuevas tecnologías del diseño. Y al mismo tiempo, un aporte desde la academia a la consolidación social del debate acerca de la identidad urbana en su sentido más amplio.

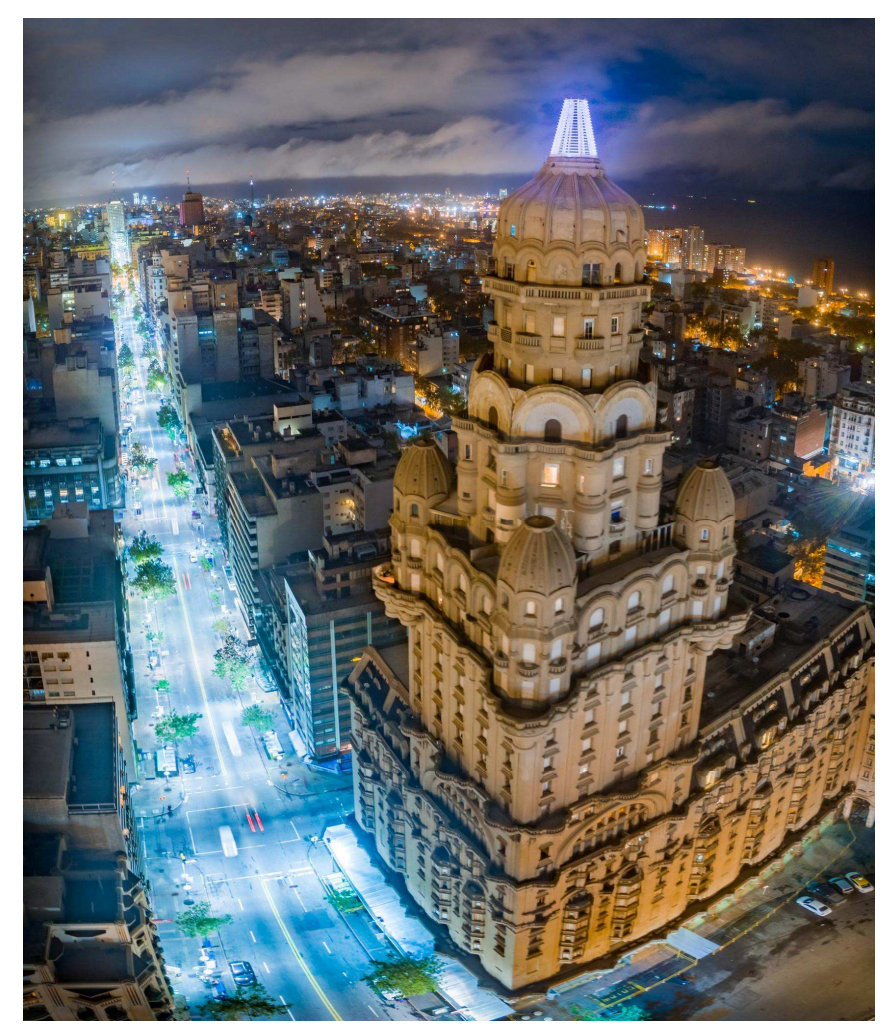

Figura 7: El Palacio Salvo actualmente, luego de la colocación del remate interactivo: linterna, faro, Gran Salvo.

\section{Equipo Gran Salvo}

Idea, Diseño, Dirección y Producción: Arq. Federico Lagomarsino. Montaje y Metalurgia: Ignacio Silva. Programación y Tecnología: Bruno Aguirre (Cuervo). Asesoramiento Estructural: Ing. Sebastián Dieste, Ing. Martín Reina (RDA Ingeniería). Asesoramiento lluminación: Ing. Ricardo Hofstadter. Equipo de Taller: Luis Blau (LCDV), Lucía Sosa, Elvis Marrero, Carolina Sánchez Panizza, Ignacio Sánchez Panizza, Inés Mir. Instalación Eléctrica:

Fernando Secinaro. Sistemas de Protección de Descargas Atmosféricas: Ing. Daniel Pereira Pierce. Seguridad e Higiene: Arq. Téc. Prev. Juan Martín Pascale. Depinfo-Vidialab-Labfab: Arq. Marcelo Payssé, MSc. Arq. Fernando García Amen. Colaborador Palacio Barolo: Arq. Fernando Carral (Argentina). 


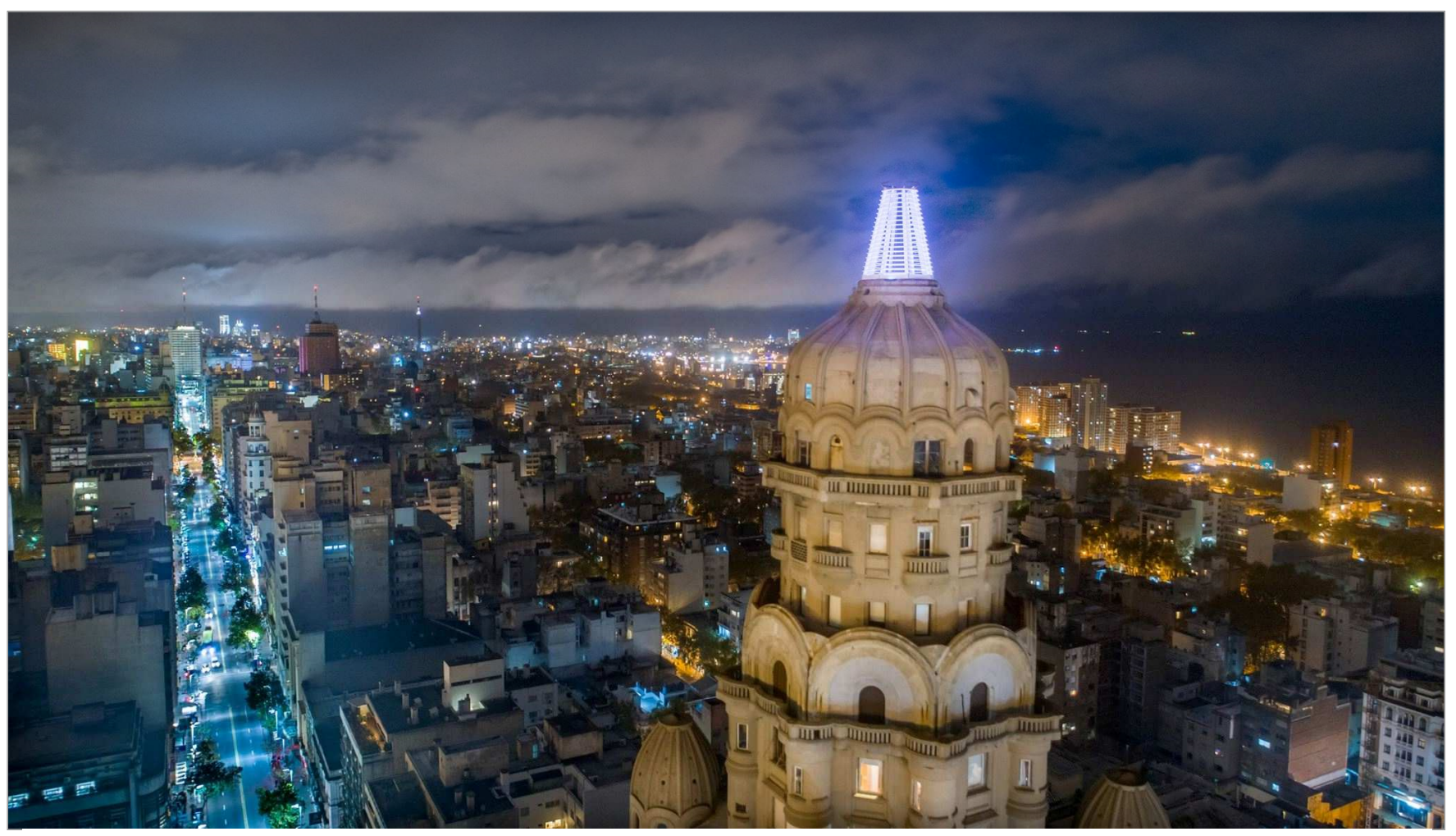

Figura 8: Nueva imagen del remate del edificio, modificando el skyline de la ciudad.

\section{Agradecimientos}

Aníbal Andrade; Carlos Varela; Alejandra Dixon; Alejandra Arseniato; Maximiliano Patrón; Carlos Sena; Mónica Kaphamme; Mantenimiento Palacio Salvo: Marcos, Jesús y Martín; Estudio Fischer Propiedad Intelectual; Alberto Quintela; Arq. Mariano Arana; Intendencia de Montevideo; Municipio B; Sociedad de Arquitectos del Uruguay; Facultad de Arquitectura, Diseño y Urbanismo de la Universidad de la Republica; Departamento de Informática Aplicada al Diseño; Vidialab; Laboratorio de Fabricación Digital; Instituto de la Construcción Facultad de Arquitectura; Arq. Fernando Islas; Andrés Dibarboure; Instituto Italiano de Cultura; Ministerio de Educación y Cultura; Ministerio de Turismo; Comisión Especial Permanente CV; Lyte Iluminación Técnica; Stiler; Hotel Radisson Victoria Plaza; Instituto Uruguayo de Normas Técnicas UNIT; Genexus; Salomón Grúas; Copiplan; Red Pagos; Neo; Sika; Cámara de la Construcción del Uruguay; La Casa del Tornillo; Sixto; Hempel -Leonortex; Sur Ascensores; Securitas; Snappy; Aluminios del Uruguay; Bancos de Seguros del Estado; SPM Equipamientos; Secinaro; Andrés Del Castillo; Drone 5; y a todos los vecinos del Palacio Salvo.

\section{Referencias}

OWAN, H.J. (1977). A history of masonry and concrete domes in building construction. Building and Environment, 12, 1-24. 2015, De Elsevier Base de datos.

GERSHENFELD, N.. (1999). The nature of mathematical modeling. Cambridge: University of Cambdridge.
LAGOMARSINO, F.; GARCIA AMEN, F. (2015). Crisálida. Revista Arquis UB, 6, 74-77.

ALBERTI, L. B. (1973) "De re aedificatoria $(1450,1485)$ ", en Opere volgari. Vol.III, Bari: Laterza.

GARCIA AMEN, F.; MARTIN IGLESIAS, R.; SCHIEDA, A.; LAGOMARSINO, F.; MIRET, S. (2016) "Digital domes that become urban symbionts", p. 892-896 In: XX Congreso de la Sociedad Iberoamericana de Gráfica Digital. São Paulo: Blucher.

CARPO, M. (2013) The Digital Turn in Architecture 1992 - 2012. London: John Wiley \& Sons.

CECCATO, C. (1999) "Microgenesis. The Architect as Toolmaker: Computer-Based Generative Design Tools and Methods", in: Soddu, C. (ed.): The Proceedings of the First International Generative Art Conference. Generative Design Lab at DiAP, Politecnico di Milano University.

EVANS, R. (1995) The Projective Cast. Architecture and its three Geometries, Cambridge: MIT Press.

FRAZER, John. An Evolutionary Architecture. London: AA Publications.

KILIAN, A., Ochsendorf, J.O. (2005) Particle-spring systems for structural form finding, in: Journal Of The International Association For Shell And Spatial Structures: IASS, Vol. 46, pp. 77-84.

KOLAREVIC, B. (2003) Architecture in the Digital Age: Design and Manufacturing. New York \& London: Spon Press - Taylor \& Francis Group.

ORTEGA, L. (2009) Digitization takes over. Barcelona. RotoVision.

PALLARES, G. (2017) Proyecto Gran Salvo Una Charla con su creador Federico Lagomarsino. Ministerio de Diseño. 